\title{
Diosgenin attenuates neuropathic pain in a rat model of chronic constriction injury
}

\author{
WEI-XIN ZHAO* ${ }^{*}$ PENG-FEI WANG ${ }^{*}$, HUI-GANG SONG and NAI SUN \\ Department of Anesthesiology, Zhengzhou Central Hospital \\ Affiliated to Zhengzhou University, Zhengzhou, Henan 450007, P.R. China
}

Received April 10, 2016; Accepted March 13, 2017

DOI: $10.3892 / \mathrm{mmr} .2017 .6723$

\begin{abstract}
Diosgenin is a steroidal saponin extract from numerous plants, including Solanum and Dioscorea species, and has been reported to possess neuroprotective activity. However, the role of diosgenin in neuropathic pain remains unclear. The present study examined the effects of diosgenin on allodynia and the levels of inflammatory mediators in rats following neuropathic pain evoked by chronic constriction injury (CCI). In addition, the underlying molecular mechanisms involved in diosgenin-induced suppression of neuropathic pain were examined. The results of the present study demonstrated diosgenin reversed CCI-decreased mechanical withdrawal threshold and thermal withdrawal latency. Furthermore, diosgenin inhibited CCI-induced upregulated levels of the pro-inflammatory cytokines tumor necrosis factor- $\alpha$, interleukin (IL)-1 $\beta$ and IL-2, and suppressed oxidative stress induced by $\mathrm{CCI}$ in the spinal cord. Furthermore, diosgenin significantly inhibited the expression of phosphorylated-p38 mitogen activated protein kinase (MAPK) and nuclear factor $(\mathrm{NF})-\kappa \mathrm{B}$ in the spinal cord in CCI rats compared with sham-operated rats. In conclusion, the present study demonstrated that diosgenin attenuates neuropathic pain in CCI rats by inhibiting activation of the $\mathrm{p} 38 \mathrm{MAPK}$ and $\mathrm{NF}-\kappa \mathrm{B}$ signaling pathways. These results implicate diosgenin in the treatment of neuropathic pain, which merits further clinical investigation.
\end{abstract}

\section{Introduction}

Neuropathic pain is a major chronic condition arising from injury or disease affecting the peripheral or central nervous system (1). It is characterized by hyperalgesia, allodynia and

Correspondence to: Dr Nai Sun, Department of Anesthesiology, Zhengzhou Central Hospital Affiliated to Zhengzhou University, 195 Tongbai Road, Zhongyuan, Zhengzhou, Henan 450007, P.R. China

E-mail: sunnai_obs@163.com

\section{${ }^{*}$ Contributed equally}

Key words: diosgenin, neuropathic pain, chronic constriction injury, inflammatory mediators spontaneous pain. Nowadays, neuropathic pain has become a significant public health problem, affecting $\sim 10-40 \%$ of the general population (2). Despite immense advances in treatment strategies, the effective treatment of patients suffering from neuropathic pain remains challenging $(3,4)$. Thus, it is urgent to investigate effective and nontoxic analgesics for the management of neuropathic pain.

Accumulating evidence has demonstrated that nerve injury-induced inflammatory cytokines and reactive oxygen species (ROS) serve important roles in the progress of neuropathic pain (5-7). Nerve damage causes the upregulation of inflammatory mediators, including tumor necrosis factor (TNF)- $\alpha$ and interleukin (IL)-1 $\beta(8,9)$. Nuclear factor $(\mathrm{NF})-\kappa \mathrm{B}$, a critical regulator of inflammatory process, has also been demonstrated to be activated in neuropathic pain (10). Therefore, inhibition of these cytokines attenuates nerve injury-induced allodynia.

Diosgenin is a steroidal saponin extract from numerous plants, including Solanum and Dioscorea species. Increasing evidences have reported that diosgenin has multiple pharmacological activities, including anti-inflammatory, anti-oxidant and anti-cancer properties (11-13). In addition, diosgenin has been reported to exert neuroprotective activity. For example, diosgenin significantly improved memory function and reduced axonal degeneration in an Alzheimer's disease mouse model (14). However, the role of diosgenin in neuropathic pain remains unclear. The present study examined the effects of diosgenin on allodynia, and the levels of inflammatory mediators in rats following neuropathic pain evoked by chronic constriction injury (CCI). In addition, the underlying molecular mechanisms involved in the diosgenin-induced suppression of neuropathic pain were investigated.

\section{Materials and methods}

Animals. Male Sprague-Dawley rats $(n=25)$ weighing 180-200 g were supplied by the Experimental Animal Centre of Zhengzhou Central Hospital Affiliated to Zhengzhou University (Zhengzhou, China). The animals were housed in a room maintained at $22 \pm 1^{\circ} \mathrm{C}$ with an alternating 12-h light/dark cycle, and provided food and water ad libitum. The animal experimental procedures were approved and reviewed by the Institutional Animal Care and Use Committee of Zhengzhou Central Hospital Affiliated to Zhengzhou University. 
Induction of neuropathic pain. Neuropathic pain was induced in experimental animals by CCI of the sciatic nerve which was performed as previously described (15). In brief, rats were anesthetized intraperitoneally with $40 \mathrm{mg} / \mathrm{kg}$ sodium pentobarbital (Sigma-Aldrich; Merck KGaA, Darmstadt, Germany). Four ligatures (silk 4-0) were tied loosely around proximal bifurcation part of the nerve with $1 \mathrm{~mm}$ spacing between each ligature, until a brisk twitch of the right hind limb was observed. Sham surgery was performed with the sciatic nerve exposed but not ligated in control rats ( $n=3$ per group).

Drug treatment. Diosgenin in doses of 10,20 and $40 \mathrm{mg} / \mathrm{kg}$ were administered intraperitoneally to neuropathic rats once a day for two weeks, starting from the first day following the induction of neuropathic pain; the sham-operated rats received normal saline $(20 \mu \mathrm{l})$ alone, following the same treatment procedure. The rats were sacrificed by spinal dislocation $24 \mathrm{~h}$ after the last administration.

Evaluation of mechanical allodynia and thermal hyperalgesia. Mechanical allodynia was evaluated as indicated by the paw withdrawal threshold in response to von Frey filaments using the up-down method according to previously described protocol (16). In brief, rats were placed in an inverted clear plexiglass cage $(23 \times 18 \times 13 \mathrm{~cm})$ on a 3 -mm-thick glass plate, and were allowed to acclimatize for $30 \mathrm{~min}$ before testing. The plantar surface of each hind paw was applied with pressure from below with the electronic Von Frey filament via the mesh floor. The force applied at the time of paw withdrawal was recorded.

Heat hypersensitivity was tested using a plantar test (cat. no. 7370; Ugo Basile Srl, Varese, Italy) according to a method described previously (17). In brief, the heat source was positioned under the glass floor directly beneath the hind paw. The heat intensity was set to last for $\sim 10 \mathrm{sec}$ to produce paw withdrawal latency, and the cut-off was set at $20 \mathrm{sec}$ to avoid tissue damage. Each paw was measured alternatively after $>5$ min.

Western blot analysis. At day 14, the rats were sacrificed by spinal dislocation. Then, the lumbar spinal cord tissues (L4/5) were rapidly removed. Proteins were extracted from the lumbar spinal cord tissues (L4/5) using RIPA Cell Lysis Buffer (Takara Biotechnology, Dalian, China). Lysates were sonicated for $5 \mathrm{sec}$ on ice and centrifuged at 6,000 x g for $10 \mathrm{~min}$ at $4^{\circ} \mathrm{C}$. Supernatants were collected and the protein concentration was quantified using a Pierce Bicinchoninic Acid Protein Assay kit (Pierce; Thermo Fisher Scientific, Inc., Waltham, MA, USA). Equal amounts of protein $(30 \mu \mathrm{g})$ were separated by $10 \%$ SDS-PAGE and subsequently transferred to polyvinylidene difluoride membranes. The membrane was blocked with $5 \%$ non-fat dry milk in Tris-buffered saline with $0.1 \%$ Tween-20 (TBST) for $1 \mathrm{~h}$ at room temperature. The membrane was then incubated with a 1:1,000 dilution of the following primary antibodies, all purchased from Santa Cruz Biotechnology Inc. (Dallas, TX, USA): rabbit anti-mouse phosphorylated (p)-p38 mitogen activated protein kinase (MAPK) antibody (sc-101759; 1:3,000), rabbit anti-mouse p38 MAPK antibody (sc-535; 1:2,500), rabbit anti-mouse p-NF- $\mathrm{B}$ p65 antibody (sc-33020; 1:3,000;) and rabbit anti-mouse GAPDH antibody (sc-25778; 1:2,500) overnight at $4{ }^{\circ} \mathrm{C}$. Following three washes with TBST buffer, the membrane was washed and incubated with a goat anti-rabbit IgG horseradish peroxidase-conjugated secondary antibody (sc-2030; 1:2,500) for $1 \mathrm{~h}$ at $37^{\circ} \mathrm{C}$. Proteins were subsequently detected by Enhanced Chemiluminescence (GE Healthcare Life Sciences, Chalfont, UK) and quantified using Gel-Pro Analyzer version 4.0 software (Media Cybernetics, Inc., Rockville, MD, USA).

Enzyme linked immunosorbent assay (ELISA). The levels of TNF- $\alpha$, IL-1 $\beta$ and IL-2 in the lumbar spinal cords were measured using commercially available rat TNF- $\alpha$ (RAB0479), IL-1 $\beta$ (RAB0277) and IL-2 (RAB0288) ELISA kits (Sigma-Aldrich; Merck KGaA) according to the manufacturer's protocol. Plates were read using an ELISA reader (Omega Bio-Tek, Inc., Norcross, GA, USA) at a wavelength of $450 \mathrm{~nm}$.

The levels of malondialdehyde (MDA) and glutathione peroxidase (GSH-PX) in the lumbar spinal cords were estimated by using MDA and GSH-PX kits from the Biological Engineering Research Institute (Nanjing, China).

Statistical analysis. Analysis was performed using SPSS 16.0 software (SPSS, Inc., Chicago, IL, USA). All data are presented as the mean \pm standard deviation. The data of behavioral tests were analyzed by two-way analysis of variance, while the data of cytokine assays were analyzed by one-way analysis of variance, followed by Newman-Keuls post hoc test. $\mathrm{P}<0.05$ were considered to indicate a statistically significant difference.

\section{Results}

Effect of diosgenin on mechanical allodynia and thermal hyperalgesia. The effects of diosgenin on mechanical allodynia and thermal hyperalgesia were examined. CCI resulted in significant development of mechanical allodynia (Fig. 1A) and thermal hyperalgesia (Fig. 1B), as compared with the sham group as assessed on day 1,7 and 14. However, diosgenin treatment reversed CCI-induced mechanical allodynia and thermal hyperalgesia in a dose-dependent manner.

Effect of diosgenin on pro-inflammatory cytokine levels in the spinal cord. There is strong evidence that pro-inflammatory cytokines have important roles in the pathology of neuropathic pain. Thus, the present study examined the effects of diosgenin on pro-inflammatory cytokine levels in spinal cord by ELISA. The levels of TNF- $\alpha$ (Fig. 2A), IL-1 $\beta$ (Fig. 2B) and IL-2 (Fig. 2C) were significantly increased in the spinal cord of CCI rats compared with the sham group. However, diosgenin reversed CCI-increased levels of TNF- $\alpha$, IL-1 $\beta$ and IL-2 in a dose-dependent manner.

Effect of diosgenin on oxidative stress in the spinal cord, following CCI. The effects of diosgenin on oxidative stress in spinal cord were examined by ELISA. Rats in the CCI group exhibited a significant increase in the production of MDA (Fig. 3A) and decrease in the content of GSH-PX (Fig. 3B), compared with the sham group. Diosgenin treatment obviously reversed CCI-induced oxidative stress in spinal cord in a dose-dependent manner.

Effect of diosgenin on p-p38 MAPK in the spinal cord, following $C C I$. It has been reported that activation of p-p38 

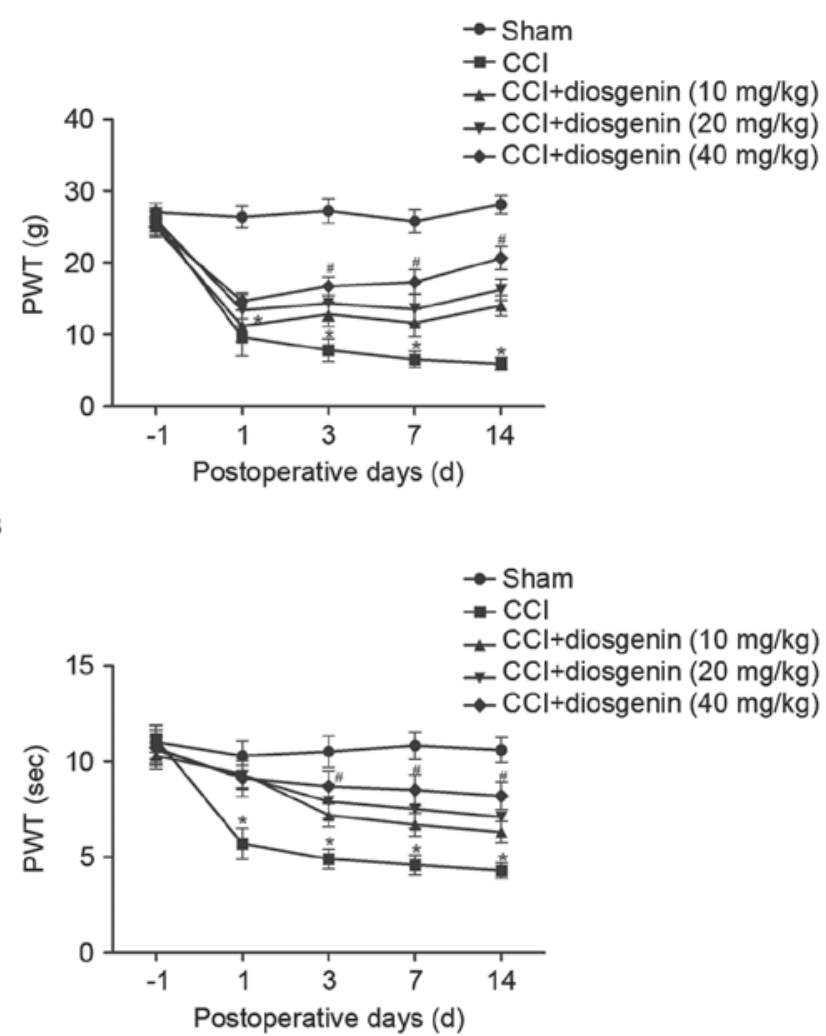

Figure 1. Effects of diosgenin on mechanical allodynia and thermal hyperalgesia. (A) PWT and (B) PWL were measured following intraperitoneal injection of 10, 20 and $40 \mathrm{mg} / \mathrm{kg}$ diosgenin. All experiments were repeated at least three times. Data are presented as the mean \pm standard deviation ( $\mathrm{n}=3$ /group). ${ }^{*} \mathrm{P}<0.05$ vs. sham group; ${ }^{*} \mathrm{P}<0.05$ vs. $\mathrm{CCI}$ group. $\mathrm{PWT}$, paw withdrawal threshold; PWL, paw withdrawal latency; CCI, chronic constriction injury.

MAPK contributes to the development of inflammatory and neuropathic pain induced by nerve injury. Therefore, the effects of diosgenin on phosphorylation of p38 MAPK in spinal cord were investigated. As presented in Fig. 4, protein expression levels of p-p38 MAPK were greatly increased by CCI, compared with the sham group. However, diosgenin treatment significantly inhibited the expression level of p-p38 MAPK in the spinal cord of CCI rats.

Effect of diosgenin on $N F-\kappa B$ activation in the spinal cord, following $C C I$. The $\mathrm{NF}-\kappa \mathrm{B}$ signaling pathway serves a key role in regulating the expression of pro-inflammatory and pain mediators. To investigate the underlying mechanism of diosgenin in CCI-induced neuropathic pain, protein expression levels of $\mathrm{p}-\mathrm{NF}-\kappa \mathrm{B}$ p 65 in spinal cord of CCI rats were detected. Western blot analysis demonstrated that the CCI group had significantly increased levels of $\mathrm{p}-\mathrm{NF}-\kappa \mathrm{B}$ p65, compared with the sham group. However, diosgenin markedly decreased the expression of $\mathrm{p}-\mathrm{NF}-\mathrm{\kappa B}$ p65 in the spinal cord of CCI rats, in a dose-dependent manner (Fig. 5).

\section{Discussion}

The present study demonstrated that diosgenin reversed CCI-decreased mechanical withdrawal threshold and thermal
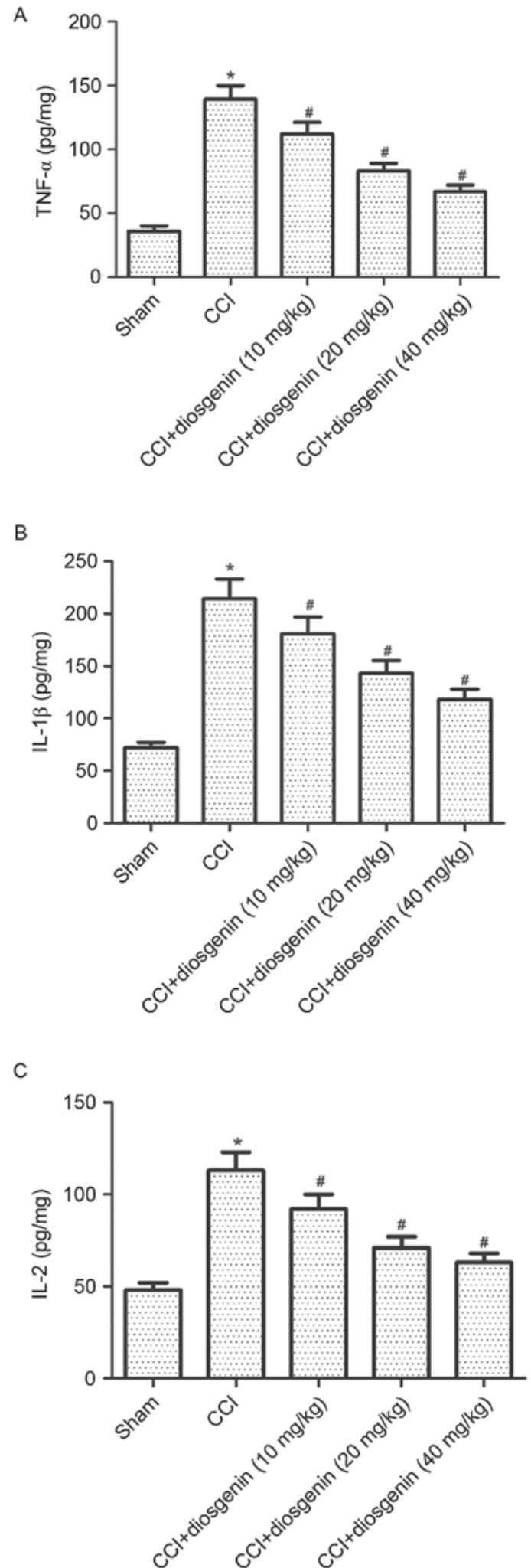

Figure 2. Effect of diosgenin on pro-inflammatory cytokine expression in the spinal cord following CCI. The expression levels of (A) TNF- $\alpha$, (B) IL-1 $\beta$ and (C) IL-2 were detected by ELISA. All experiments were repeated at least three times. Data are presented as the mean \pm standard deviation ( $n=3 /$ group). ${ }^{*} \mathrm{P}<0.05$ vs. sham group; ${ }^{\#} \mathrm{P}<0.05$ vs. CCI group. TNF- $\alpha$, tumor necrosis factor- $\alpha$; IL, interleukin; CCI, chronic constriction injury.

withdrawal latency. Diosgenin inhibited CCI-induced increased levels of the pro-inflammatory cytokines TNF- $\alpha$, IL-1 $\beta$ and IL-2, and suppressed oxidative stress induced by 
A

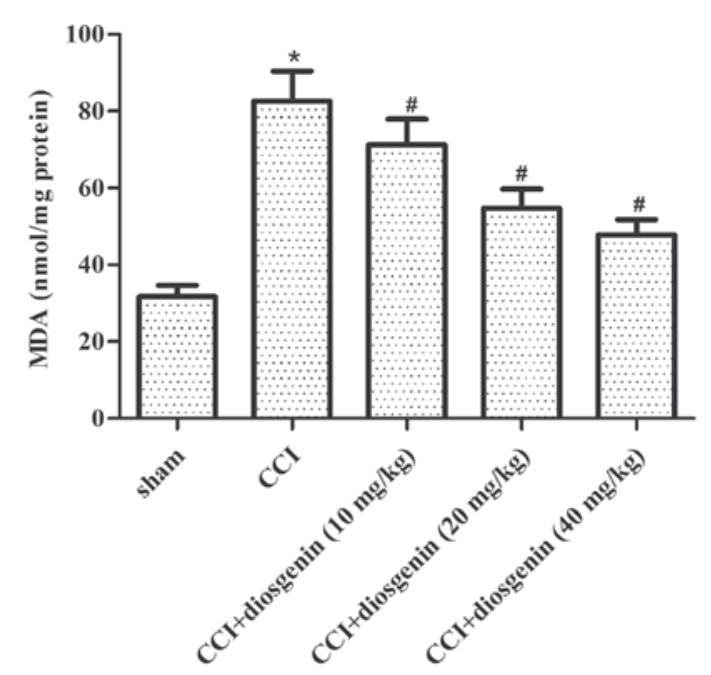

B

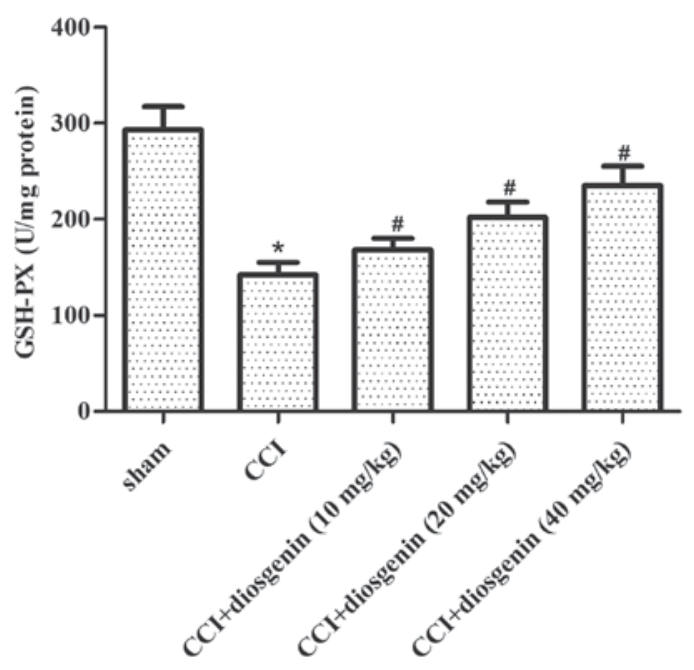

Figure 3. Effect of diosgenin on oxidative stress in the spinal cord following CCI. The rats were sacrificed $24 \mathrm{~h}$ after the last administration. The levels of (A) MDA and (B) GSH-PX in the lumbar spinal cords were estimated using MDA and GSH-PX kits. All experiments were repeated at least three times. Data are presented as the mean \pm standard deviation ( $\mathrm{n}=3$ /group). ${ }^{*} \mathrm{P}<0.05$ vs. sham group; ${ }^{\#} \mathrm{P}<0.05$ vs. CCI group. CCI, chronic constriction injury; MDA, malondialdehyde; GSH-PX, glutathione peroxidase.

$\mathrm{CCI}$ in the spinal cord. Furthermore, diosgenin significantly inhibited protein expression levels of p-p38 MAPK and NF- $\mathrm{kB}$ in the spinal cord induced by CCI.

The CCI model is the most commonly employed neuropathic pain model of nerve damage-induced allodynia/hyperalgesia (18). The present study constructed the CCI model to investigate the effects of diosgenin on allodynia/hyperalgesia and the levels of inflammatory mediators in rats following neuropathic pain. It was observed that CCI led to significant development of mechanical allodynia and heat hyperalgesia following surgery. However, diosgenin reversed CCI-induced mechanical allodynia and thermal hyperalgesia in a dose-dependent manner. These data suggested that diosgenin may attenuate neuropathic pain in a CCI model.
A
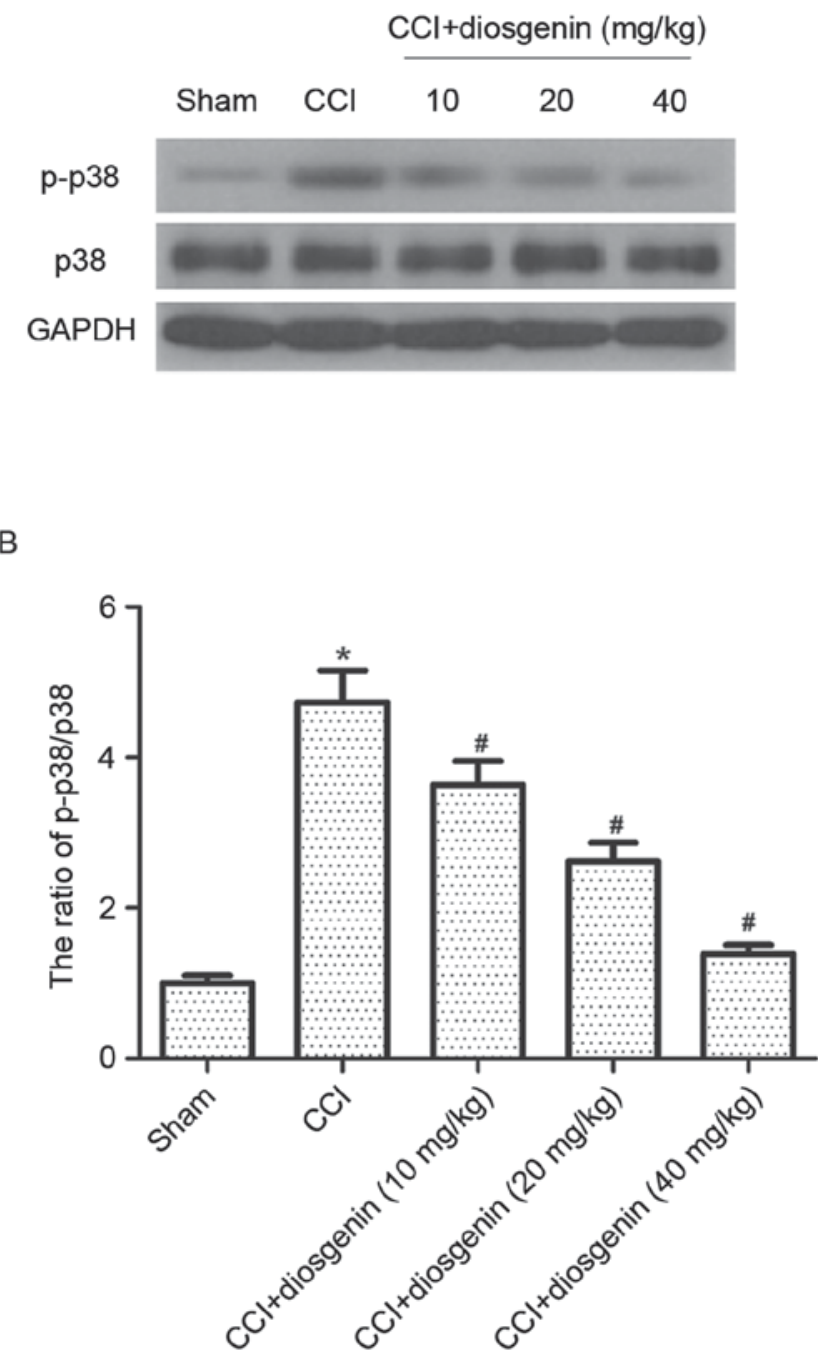

Figure 4. Effect of diosgenin on p-p38 MAPK expression levels in the spinal cord following CCI. (A) Representative western blot images and (B) quantification of protein expression levels of p-p38 MAPK in the spinal cord. GAPDH served as a loading control. All experiments were repeated at least three times. Data are presented as the mean \pm standard deviation (n=3/group). ${ }^{*} \mathrm{P}<0.05$ vs. sham group; ${ }^{*} \mathrm{P}<0.05$ vs. $\mathrm{CCI}$ group. $\mathrm{CCI}$, chronic constriction injury; MAPK, mitogen activated protein kinase; $p$, phosphorylated.

Increasing evidence suggests that peripheral nerve injury contributes to neuropathic pain via upregulation of pro-inflammatory cytokines (19-21). TNF- $\alpha$ is a predominant pro-inflammatory cytokine contributing to pain hypersensitivity following nerve damage; intrathecal injection of a TNF- $\alpha$ inhibitor prior to nerve injury reduces neuropathology and pain-associated behaviors (22). In addition, IL-1 $\beta$ levels increase significantly in the sciatic nerve following CCI (23). Consistent with previous studies, the present study demonstrated that the levels of TNF- $\alpha$, IL-1 $\beta$ and IL-2 were significantly increased in the spinal cord of CCI rats, compared sham-operated rats. However, diosgenin treatment reversed this effect in a dose-dependent manner. These results suggested that the beneficial effects of diosgenin in CCI-induced neuropathic pain are mediated via its attenuating effect on pro-inflammatory mediators.

Previous studies have indicated that CCI produces significant oxidative damage in the sciatic nerve due to the 
A

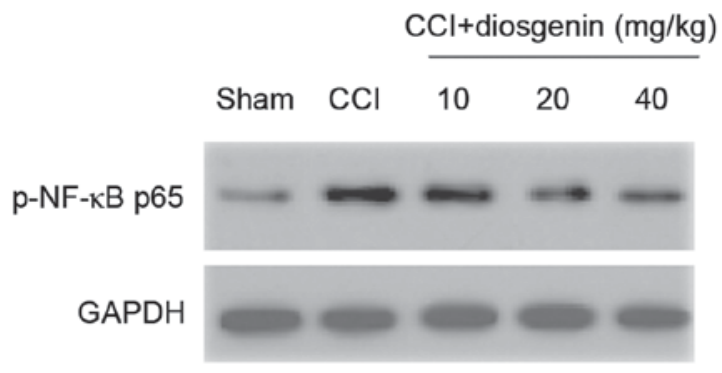

B

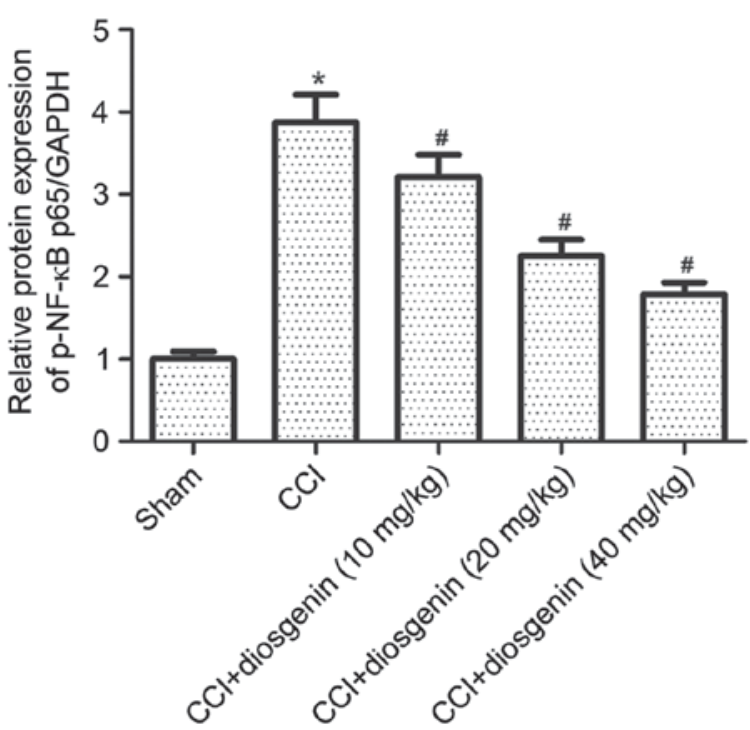

Figure 5. Effect of diosgenin on $N F-\kappa B$ activation in the spinal cord following CCI. (A) Representative western blot images and (B) quantification of $\mathrm{p}-\mathrm{NF}-\kappa \mathrm{B}$ p 65 protein expression levels in the spinal cord. GAPDH served as a loading control. Data are presented as the mean \pm standard deviation ( $\mathrm{n}=3$ /group). ${ }^{*} \mathrm{P}<0.05$ vs. sham group; ${ }^{\#} \mathrm{P}<0.05$ vs. $\mathrm{CCI}$ group. $\mathrm{CCI}$, chronic constriction injury; NF- $\kappa \mathrm{B}$, nuclear factor- $\kappa \mathrm{B}$; $\mathrm{p}$, phosphorylated.

increase in lipid peroxidation and ROS concentration $(7,24)$. Administration of natural and synthetic ROS scavengers reduces allodynia and hyperalgesia in a number of neuropathic pain models $(25,26)$. The present study revealed that $\mathrm{CCI}$ resulted in a significant increase in the production of MDA, and a decrease in the content of GSH-PX. However, diosgenin treatment reversed the CCI-induced oxidative stress in the spinal cord. These results suggested that the beneficial effects of diosgenin in CCI-induced neuropathic pain are mediated via its attenuating effect on oxidative stress.

Previous studies have demonstrated that p-p38 MAPK in spinal cord glial cells after peripheral nerve injury are involved in the development of neuropathic pain (27-29). Tsuda et al (30) reported that administration of a p38 MAPK inhibitor attenuates the development of nerve injury-induced tactile allodynia. Furthermore, the NF- $\kappa \mathrm{B}$ signaling pathway has been implicated in the mediation of neuropathic pain (31-33). Intrathecal infusion of the $\mathrm{NF}-\kappa \mathrm{B}$ inhibitor ammonium pyrolidine dithiocarbamate improved mechanical allodynia and downregulated the overexpression of TNF- $\alpha$ induced by peri-sciatic administration of TNF (34). The present study revealed that diosgenin significantly inhibited CCI-induced upregulated expression levels of p-p38 MAPK and p-NF- $\mathrm{B}$ p65 in the spinal cord. These data suggested that diosgenin attenuates neuropathic pain in CCI rats by inhibiting activation of the p38 MAPK and NF- $\kappa$ B signaling pathways.

In conclusion, the present study demonstrated that diosgenin may be effective to reduce neuropathic pain by inhibition of activation of the $\mathrm{p} 38 \mathrm{MAPK}$ and $\mathrm{NF}-\kappa \mathrm{B}$ signaling pathways. These results implicate diosgenin in the treatment of neuropathic pain, which merits further clinical investigation.

\section{References}

1. Rowbotham MC: Mechanisms of neuropathic pain and their implications for the design of clinical trials. Neurology 65 (12 Suppl 4): S66-S73, 2005.

2. Neville A, Peleg R, Singer Y, Sherf M and Shvartzman P: Chronic pain: A population-based study. Isr Med Assoc J 10: 676-680, 2008 .

3. Baastrup C and Finnerup NB: Pharmacological management of neuropathic pain following spinal cord injury. CNS Drugs 22: 455-475, 2008.

4. Finnerup NB, Otto M, McQuay H, Jensen TS and Sindrup SH: Algorithm for neuropathic pain treatment: An evidence based proposal. Pain 118: 289-305, 2005.

5. Dray A: Inflammatory mediators of pain. Brit J Anaesth 75: 125-131, 1995.

6. DeLeo JA and Yezierski RP: The role of neuroinflammation and neuroimmune activation in persistent pain. Pain 90: 1-6, 2001.

7. Kim HK, Park SK, Zhou JL, Taglialatela G, Chung K, Coggeshall RE and Chung JM: Reactive oxygen species (ROS) play an important role in a rat model of neuropathic pain. Pain 111: 116-124, 2004.

8. Ohtori S, Takahashi K, Moriya H and Myers RR: TNF-alpha and TNF-alpha receptor type 1 upregulation in glia and neurons after peripheral nerve injury: Studies in murine DRG and spinal cord. Spine (Phila Pa 1976) 29: 1082-1088, 2004.

9. Taves S, Berta T, Chen G and Ji RR: Microglia and spinal cord synaptic plasticity in persistent pain. Neural Plast 2013: 753656 , 2013.

10. Ma W and Bisby MA: Increased activation of nuclear factor kappa B in rat lumbar dorsal root ganglion neurons following partial sciatic nerve injuries. Brain Res 797: 243-254, 1998.

11. Jung DH, Park HJ, Byun HE, Park YM, Kim TW, Kim BO, Um SH and Pyo S: Diosgenin inhibits macrophage-derived inflammatory mediators through downregulation of CK2, JNK, NF-kappaB and AP-1 activation. Int Immunopharmacol 10: 1047-1054, 2010.

12. Son IS, Kim JH, Sohn HY, Son KH, Kim JS and Kwon CS: Antioxidative and hypolipidemic effects of diosgenin, a steroidal saponin of yam (Dioscorea spp.), on high-cholesterol fed rats. Biosci Biotechnol Biochem 71: 3063-3071, 2007.

13. Moalic S, Liagre B, Corbière C, Bianchi A, Dauça M, Bordji K and Beneytout JL: A plant steroid, diosgenin, induces apoptosis, cell cycle arrest and COX activity in osteosarcoma cells. FEBS Lett 506: 225-230, 2001.

14. Tohda C, Urano T, Umezaki M, Nemere I and Kuboyama T: Diosgenin is an exogenous activator of 1, 25D3-MARRS/ Pdia3/ERp57 and improves Alzheimer's disease pathologies in 5XFAD mice. Sci Rep 2: 535, 2012.

15. Bennett GJ and Xie YK: A peripheral mononeuropathy in rat that produces disorders of pain sensation like those seen in man. Pain 33: 87-107, 1988.

16. Chaplan SR, Bach FW, Pogrel JM, Chung JM and Yaksh TL: Quantitative assessment of tactile allodynia in the rat paw. J Neurosci Methods 53: 55-63, 1994.

17. Hargreaves K, Dubner R, Brown F, Flores C and Joris J: A new and sensitive method for measuring thermal nociception in cutaneous hyperalgesia. Pain 32: 77-88, 1988.

18. Jaggi AS, Jain V and Singh N: Animal models of neuropathic pain. Fund Clin Pharmacol 25: 1-28, 2011.

19. Nadeau S, Filali M, Zhang J, Kerr BJ, Rivest S, Soulet D, Iwakura Y, de Rivero Vaccari JP, Keane RW and Lacroix S: Functional recovery after peripheral nerve injury is dependent on the pro-inflammatory cytokines IL-1 $\beta$ and TNF: Implications for neuropathic pain. J Neurosci 31: 12533-12542, 2011. 
20. Detloff MR, Fisher LC, McGaughy V, Longbrake EE, Popovich PG and Basso DM: Remote activation of microglia and pro-inflammatory cytokines predict the onset and severity of below-level neuropathic pain after spinal cord injury in rats. Exp Neurol 212: 337-347, 2008

21. Zhang JM and An J: Cytokines, inflammation, and pain. Int Anesthesiol Clin 45: 27-37, 2007.

22. Zanella JM, Burright EN, Hildebrand K, Hobot C, Cox M, Christoferson L and McKay WF: Effect of etanercept, a tumor necrosis factor-alpha inhibitor, on neuropathic pain in the rat chronic constriction injury model. Spine (Phila Pa 1976) 33: 227-234, 2008

23. del Rey A, Yau HJ, Randolf A, Centeno MV, Wildmann J, Martina M, Besedovsky HO and Apkarian AV: Chronic neuropathic pain-like behavior correlates with IL-1 $\beta$ expression and disrupts cytokine interactions in the hippocampus. Pain 152: 2827-2835, 2011

24. Park ES, Gao X, Chung JM and Chung K: Levels of mitochondrial reactive oxygen species increase in rat neuropathic spinal dorsal horn neurons. Neurosci Lett 391: 108-111, 2006.

25. Navarro SA, Serafim KG, Mizokami SS, Hohmann MS, Casagrande R and Verri WA Jr: Analgesic activity of piracetam: Effect on cytokine production and oxidative stress. Pharmacol Biochem Behav 105: 183-192, 2013.

26. Twining CM, Sloane EM, Milligan ED, Chacur M, Martin D, Poole S, Marsh H, Maier SF and Watkins LR: Peri-sciatic proinflammatory cytokines, reactive oxygen species and complement induce mirror-image neuropathic pain in rats. Pain 110: 299-309, 2004.

27. Ji RR and Suter MR: p38 MAPK, microglial signaling, and neuropathic pain. Mol Pain 3: 33, 2007.
28. Zhuang ZY, Kawasaki Y, Tan PH, Wen YR, Huang J and Ji RR: Role of the CX3CR1/p38 MAPK pathway in spinal microglia for the development of neuropathic pain following nerve injury-induced cleavage of fractalkine. Brain Behav Immun 21: 642-651, 2007.

29. Hua XY, Svensson CI, Matsui T, Fitzsimmons B, Yaksh TL and Webb M: Intrathecal minocycline attenuates peripheral inflammation-induced hyperalgesia by inhibiting p38 MAPK in spinal microglia. Eur J Neurosci 22: 2431-2440, 2005.

30. Tsuda M, Mizokoshi A, Shigemoto-Mogami Y, Koizumi S and Inoue K: Activation of p38 mitogen-activated protein kinase in spinal hyperactive microglia contributes to pain hypersensitivity following peripheral nerve injury. Glia 45: 89-95, 2004.

31. Sun T, Song WG, Fu ZJ, Liu ZH, Liu YM and Yao SL: Alleviation of neuropathic pain by intrathecal injection of antisense oligonucleotides to p65 subunit of NF-kappaB. Brit J Anaesth 97: 553-558, 2006.

32. Tegeder I, Niederberger E, Schmidt R, Kunz S, Gühring H, Ritzeler O, Michaelis M and Geisslinger G: Specific inhibition of IkappaB kinase reduces hyperalgesia in inflammatory and neuropathic pain models in rats. J Neurosci 24: 1637-1645, 2004

33. Lee KM, Jeon SM and Cho HJ: Tumor necrosis factor receptor 1 induces interleukin-6 upregulation through NF-kappaB in a rat neuropathic pain model. Eur J Pain 13: 794-806, 2009.

34. Wei XH, Yang T, Wu Q, Xin WJ, Wu JL, Wang YQ, Zang Y, Wang J, Li YY and Liu XG: Peri-sciatic administration of recombinant rat IL-1 $\beta$ induces mechanical allodynia by activation of src-family kinases in spinal microglia in rats. Exp Neurol 234: 389-397, 2012. 